\title{
Caveolin Expression is Common among Benign and Malignant Smooth Muscle and Adipocyte Neoplasms
}

Ilene Bayer-Garner, M.D., Michael Morgan, M.D., Bruce R. Smoller, M.D.

Departments of Pathology (IB-G, BRS) and Dermatology (BRS), University of Arkansas for Medical Sciences, Little Rock, Arkansas; and Department of Pathology (MM), James Haley Veterans Administration

Hospital, Tampa, Florida

The caveolins belong to a newly described group of membrane-scaffolding proteins. Their presence in benign endothelial cells has been used to discriminate benign from malignant vascular neoplasms. The extent of caveolin expression in cutaneous mesenchymal neoplasms has not yet been evaluated, and thus the diagnostic utility of these antibodies is not yet known. In our study, we immunohistochemically examined a spectrum of tumors derived from smooth muscle and adipocytes for caveolin expression. We found that both benign and malignant smooth muscle tumors and tumors comprised of adipocytes expressed caveolins. The presence of this protein in a range of mesenchymal neoplasms is important to know about as this decreases the reported specificity of a positive finding. It is doubtful that caveolin down-regulation contributes to the pathogenesis of liposarcomas and leiomyosarcomas. This finding also may suggest a common origin between endothelial cells and other mesenchymal cells.

KEY WORDS: Angiogenesis inhibition; Caveolin; Membrane-scaffolding protein; Mesenchymal neoplasm; Vascular neoplasm.

Mod Pathol 2002;15(1):1-5

The caveolins belong to a group of structural proteins that have been described in the endothelial cells and adipocytes of benign mesenchymal tissue (1). They appear to play a central role in forming vesicular invaginations that regulate various signal transduction proteins within these cells (1). Caveolins may also play a role in inhibiting angiogenesis. It has been proposed that down-regulation of the caveolins may play a role in neovascularization and

Copyright (C) 2002 by The United States and Canadian Academy of Pathology, Inc

VOL. 15, NO. 1, P. 1, 2002 Printed in the U.S.A.

Date of acceptance: August 30, 2001.

Address reprint requests to: Bruce R. Smoller, M.D., Department of Pathology, Slot 517, University of Arkansas for Medical Sciences, 4301 W. Markham, Little Rock, AR 72205; e-mail: smollerbrucer@uams.edu. in the development of metastatic disease (2-4). Caveolin expression has been described in a range of vascular neoplasms, including lobular capillary hemangiomas, targetoid hemosiderotic hemangiomas, and tufted angiomas (5). In the same study, decreased expression of this protein was detected in angiosarcomas, Kaposi's sarcoma, and epithelioid hemangioendotheliomas (5). These results suggest that anti-caveolin antibodies may play a useful role in attempting to distinguish benign from malignant vascular neoplasms.

Some authors have described caveolin expression in benign adipocytes (1), but this finding has not been replicated in all studies (5). However, neoplasms derived from (or differentiating toward) other types of mesenchymal cells have not been studied. Because the differential diagnosis of mesenchymal neoplasms in the skin often includes tumors of smooth muscle cells and adipocytes, we examined a series of these types of neoplasms for the expression of this cytoplasmic protein. Differential expression of this protein may prove to be diagnostically helpful in further distinguishing between these histologically similar neoplasms.

\section{MATERIALS AND METHODS}

Biopsies of soft-tissue neoplasms were obtained from the pathology tissue files at the University of Arkansas for Medical Sciences (UAMS) between 1997 and 2000. The lesions consisted of ten intrauterine leiomyomas, twenty-three lipomas (one cutaneous lipoma, eight deep lipomas, one chondroid lipoma, two spindle cell lipomas, four angiomyolipomas, six angiolipomas, and one thymolipoma), sixteen leiomyosarcomas (six high grade, three intermediate grade, one low grade, two myxoid, two metastatic, and two which were not graded), and twenty-eight liposarcomas (nine high grade, four intermediate grade, fifteen low grade). The single cutaneous lipoma was located on the forehead, the deep lipomas were located in the soft tissue of the back and extremities, the angiomyolipomas were 
all located in kidneys, and the angiolipomas were located in the soft tissue and skin of the upper extremities, with a single case located in the soft tissue of the abdominal wall. The leiomyosarcomas were located in the uterus (seven), the vena cava (two), and a single case was located in each of the following: maxillary sinus, stomach, vagina, rectum, pancreas, lung, and pelvis. The liposarcomas were located in the upper extremity (seven), the lower extremity (fifteen), the groin (three), the small bowel (two), and the chest wall (one). Hematoxylin and eosin (H\&E)-stained sections were diagnosed by various staff pathologists at UAMS. These diagnoses were confirmed by one of us (BRS) before initiating the study.

All specimens were fixed in $10 \%$ formalin and embedded in paraffin. Three- $\mu \mathrm{m}$ sections were cut and mounted on 3-aminopropyltrethoxy-silane (APS)-coated slides, dried, and deparaffinized before undergoing antigen retrieval by heat treatment, via a decloaking chamber (Biocare, Walnut Creek, CA), in DAKO Target Retrieval solution (DAKO, Carpenteria, CA). Immunohistochemical staining was performed with the DAKO autostainer system (DAKO) for all steps from endogenous peroxidase blocking through the application and development of the chromogenic substrate. The DAKO LSAB + Kit was used with a 1:100 dilution of anti-caveolin (Transduction Laboratories, Lexington $\mathrm{KY}$ ), an $\mathrm{IgG}_{1}$ mouse anti-human antibody. Sections were incubated with biotinylated secondary antibody for fifteen minutes, and then with streptavidin alkaline phosphatase for fifteen minutes. The signal was visualized with 3,3'-diaminobenzidine in chromogen solution, which yielded a brown product at the site of the target antigen. Slides were counterstained with hematoxylin for thirty seconds. The positive internal control consisted of vascular structures, which showed cytoplasmic membrane staining. The negative control consisted of a 1:100 dilution of a nonspecific $\operatorname{IgG}_{1}$ mouse antibody (DAKO) that was substituted for anti-caveolin. The distribution of immunoreactivity to anti-caveolin was analyzed (by IBBG and BRS) by quantifying the cytoplasmic membrane staining for each case. Positivity was defined as those cases in which over $90 \%$ of the neoplastic cells expressed cytoplasmic immunoreactivty for anti-caveolin.

\section{RESULTS}

Evaluation of H\&E-stained slides by light microscopy showed typical histologic features of leiomyomas, lipomas, leiomyosarcomas, and liposarcomas. Immunoreactivity for anti-caveolin was demonstrated in the muscle, adipose, and vascular tissue of all the neoplasms, whereas immunohistochemi- cal staining was absent in all sections stained with an isotype-matched control antibody. Anti-caveolin expression was also evaluated in nonneoplastic tissue and revealed consistent similar expression in adipose, muscle, and vascular tissue. Normal endometrial glands and stroma as well as renal tubules did not show anti-caveolin reactivity. Small bowel epithelium also showed no anti-caveolin immunoreactivity; however, the smooth muscle, muscularis propria, and vascular structures showed anticaveolin staining. Interestingly, the epidermis showed only basal layer immunoreactivity for anticaveolin, with the remainder of the epidermis showing no staining whatsoever.

Anti-caveolin staining was consistently expressed in all neoplasms of muscle, adipose, and vascular derivation. The leiomyomas showed strong diffuse anti-caveolin staining of the myocyte nucleus and cytoplasm and showed moderate staining of the endothelial cell nucleus and cytoplasm, whereas fibroblasts showed no staining (Fig. 1). Cutaneous and deep lipomas and chondroid lipomas showed diffuse peripheral adipocyte membrane staining, with focal nuclear membrane staining (Fig. 2). Spindle cell lipomas showed strong membrane staining in vascular tissue and well-differentiated adipocytes, whereas the densely cellular spindle-cell foci showed no anti-caveolin immunoreactivity. Angiomyolipomas showed strong diffuse adipocyte membrane immunopositivity for anti-caveolin as well as membrane staining in vascular endothelium, vascular smooth muscle, and extravascular muscle (Fig. 3). The angiolipomas showed similar adipocyte membrane staining, but the endothelial vascular membrane staining was greater than that of the adipocytes. A single thymolipoma showed no staining of Hassle's corpuscles and diffuse adipoctye membrane anti-caveolin immunopositivity, as encountered with the other lipomas.

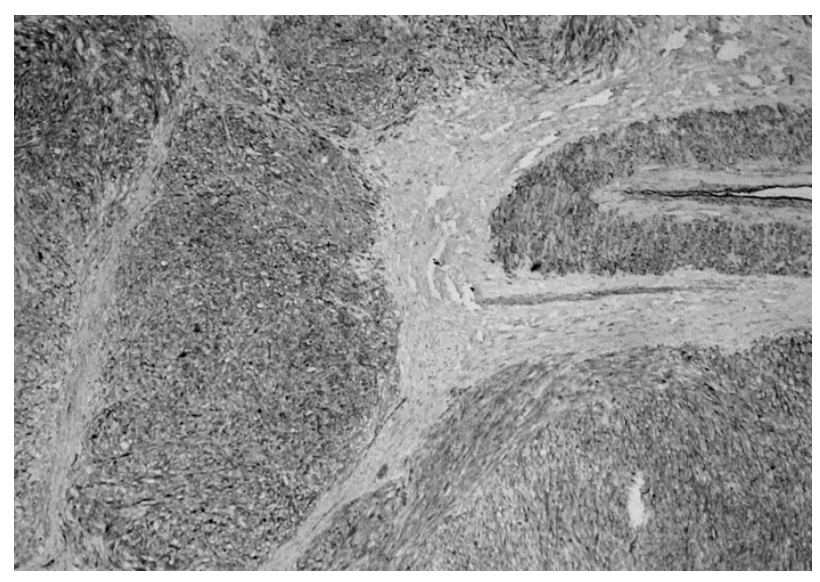

FIGURE 1. A leiomyoma with strong diffuse anti-caveolin staining in tissue of the myocyte nucleus and cytoplasm, whereas fibroblasts showed no staining. Note the strong caveolin staining in muscle and endothelial layer of the artery in the upper right-hand corner $(40 \times)$. 


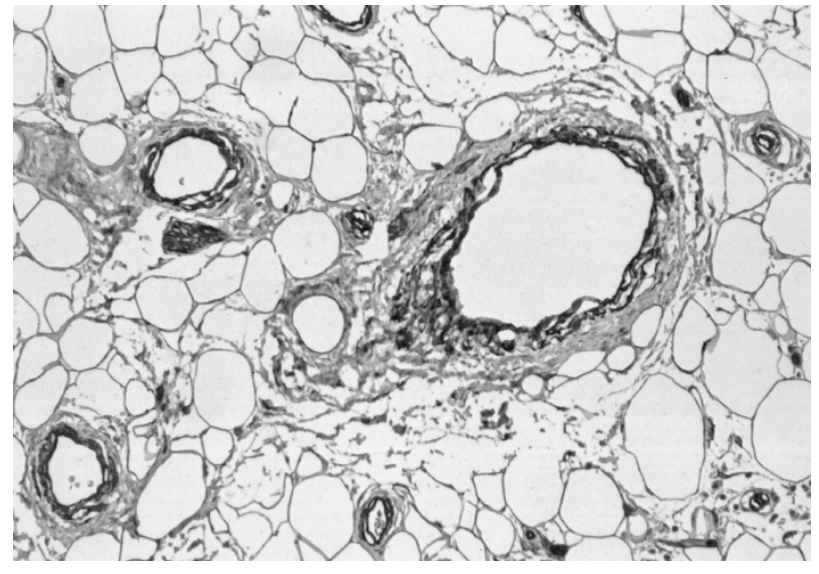

FIGURE 2. A lipoma with diffuse caveolin staining of the peripheral adipocyte membrane and focal nuclear membrane staining. Note the strong caveolin staining of the vascular structures $(40 \times)$.

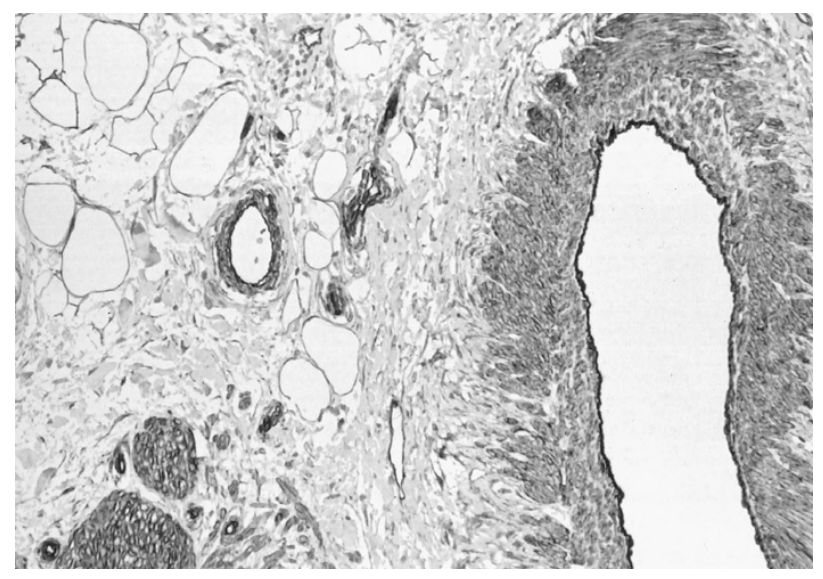

FIGURE 3. Angiomyolipomas with strong diffuse adipocyte membrane immunopositivity for caveolin as well as staining in both the vascular and muscle tissue $(40 \times)$.

Leiomyosarcomas demonstrated anti-caveolin immunoreactivity of muscle-derived tissue that was associated with the level of differentiation, in that well-differentiated areas showed strong staining (Fig. 4), whereas less differentiated areas showed weaker to absent staining (not shown). The epithelioid variants showed less immunoreactivity, whereas necrotic areas showed no anti-caveolin immunoreactivity. Liposarcomas showed a staining pattern that was also associated with level of differentiation in that welldifferentiated areas showed staining similar to that seen in lipomas with diffuse membrane adipocyte staining (Fig. 5), whereas less differentiated areas showed weak to absent staining. There was no difference in anti-caveolin staining with respect to subtypes of leiomyosarcomas including myxoid, round cell, and pleomorphic subtypes. The patient's age and gender did not influence the immunohistochemical results.

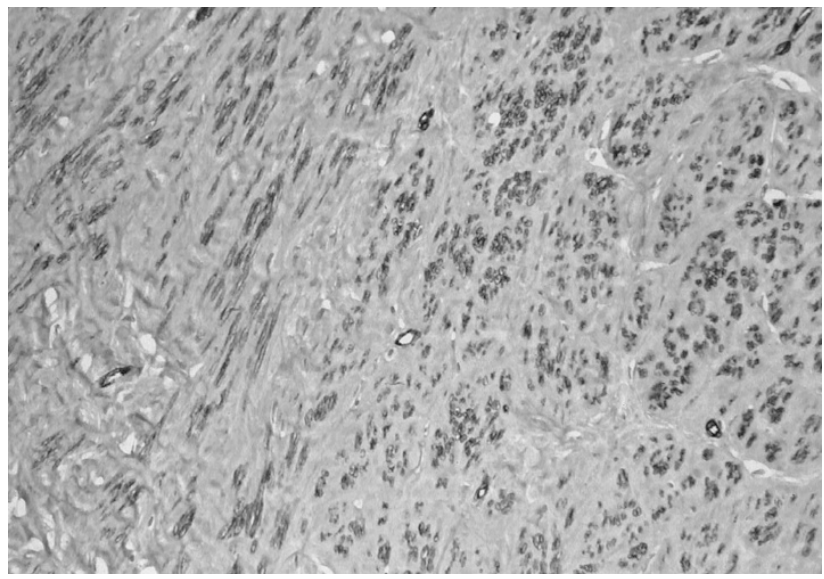

FIGURE 4. Leiomyosarcomas with caveolin immunoreactivity of muscle-derived tissue $(40 \times)$.

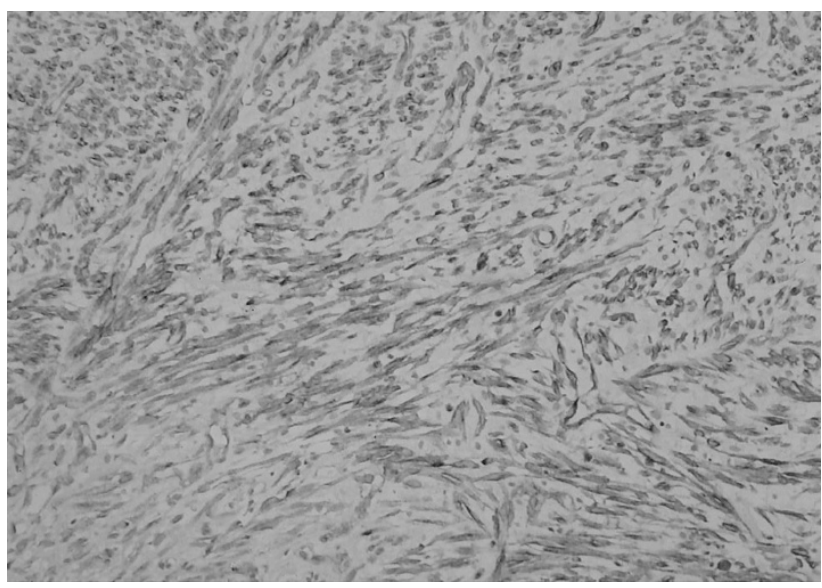

FIGURE 5. A liposarcomas with diffuse membrane adipocyte staining $(40 \times)$.

\section{DISCUSSION}

Immunohistochemical analysis of the scaffolding protein caveolin failed to discriminate among a series of benign and well-differentiated smooth muscle and adipocyte neoplasms. Caveolin immunostaining was identified in both the nucleus and cytoplasm of cells that comprised a variety of mesenchymal tumors, including the adipocyte of all types of lipoma and well-differentiated liposarcoma, the myocyte of angiomyolipoma, leiomyoma, and well-differentiated leiomyosarcoma. The immunostaining properties were uniform among the cells of each of these lesions, and gender and age did not influence the results. All of the poorly differentiated and dedifferentiated sarcoma, including leiomyosarcoma and liposarcoma, showed weak immunoreactivity or failed to stain with caveolin. These observations may have important pathogenic implications.

Very little is known regarding the pathogenesis of smooth muscle and adipocyte transformation. An established role for the Epstein-Barr virus (EBV) in 
the pathogenesis of leiomyosarcoma has been shown among immunodeficient patients (6). To the contrary, latent EBV infection is not thought to play a pathogenic role in leiomyosarcoma among immunocompetent individuals, nor is it thought to contribute to the pathogenesis of leiomyoma (7). Acquired alterations in the tumor suppressor p53 and retinoblastoma (RB) genes have been repeatedly shown in leiomyosarcoma, thus implicating a pathogenic role for these tumor suppressor genes in myocyte transformation $(8,9)$. These findings are underscored by the recent demonstration of consistent cytogenetic aberrations within these tumors, and the predictable existence of altered genomic sequences encoding important oncogenes and tumor suppressor foci. Recurrent alterations involving deletions of the long arm of the 7th chromosome (7) (q11.2-22q31-32) and translocation of the 12th and 14th chromosomes $\mathrm{t}(12,14)$ (q14-15; q2324) have been shown in leiomyoma with diverse and complex rearrangements identified within leiomyosarcoma (10-12). Progressive and reproducible cytogenetic alterations have also been shown among lipomas, atypical lipomas, and liposarcomas. It has been postulated that these progressive genetic alterations are responsible for their pathogenesis and are manifest in the phenotypic continuum observed with these tumors (13-17). For instance, lipomas at the benign end of the spectrum show 12q13-15 rearrangements, atypical lipomas show both a complex 12q13-15 rearrangement with isochromosome (ring) formation and 12q13-15 amplification, and finally, liposarcomas at the other end of the spectrum show translocation of the 12th and 16th chromosomes, $t(12,16)(q 13 ; p 11)$. The 12q13-15 chromosome region encodes for a series of important cell cycle kinase and tumor suppressor genes, including CDK4, the high-mobility group proteins (HMGs), and the p53 inhibitor MDM-2 (18, 19). An essential function of caveolin is to directly interact with and regulate G-proteins and Ha-ras tyrosine kinase expression $(3,4)$. Both of these proteins possess signal-transducing properties that ultimately bind to both CDKs and HMGs regulating RNA transcription and thus controlling the cell cycle (1). Given the regulatory properties of caveolin and understanding that the genes encoded on the region of the altered 12th chromosome increasingly renders them capable of circumventing regulation, it is plausible that down-regulation or loss of caveolin contributes to or follows sarcomatous dedifferentiation as observed in this study. Furthermore, as antibodies to caveolin failed to stain the poorly differentiated or dedifferentiated sarcomas, it is possible that down-regulation of caveolin may represent a latent alteration permissive to or characteristic of tumor dedifferentiation. Interestingly, there was no difference in the immunolabeling in- tensity observed between the benign mesenchymal tumors and well-differentiated sarcomas. Our current findings differ slightly with a previous observation of an earlier detectable loss of caveolin expression among vascular-derived tumors of low malignant potential (5). In the earlier study, there was a stepwise decrement in caveolin expression observed between benign vascular tumors such as lobular capillary hemangiomas, tumors of intermediate malignant potential such as hemangioendothelioma, and tumors of aggressive potential such as angiosarcoma. Determinants responsible for the differential properties of expression among mesenchymal tumors are yet unknown but could be due to inherently different levels of caveolin expression within endogenous tissues (1) or to differential regulation of expression by mitigating cellular influences within or outside the neoplastic cells.

\section{REFERENCES}

1. Okamoto T, Schlegel A, Scherer P, Scherer PE, Lisanti MP. Caveolins, a family of scaffolding proteins for organizing "preassembled signaling complexes" at the plasma membrane. J Biol Chem 1998;273:5419-22.

2. Liu J, Razani B, Tang S, Terman BI, Ware JA, Lisanti MP. Angiogenesis activators and inhibitors differentially regulate caveolin expression and caveola formation in vascular endothelial cells. Angiogenesis inhibitors block vascular endothelial growth factor-induced down-regulation of caveolin-1. J Biol Chem 1999;274:15781-5.

3. Engelman J, Wykoff C, Yasuhara S, Song KS, Okamoto T, Lisanti MP. Recombinant expression of caveolin-1 in oncogenically transformed cells abrogates anchorage-independent growth. J Biol Chem 1997;272:16374-81.

4. Koleske A, Baltimore D, Lisanti MP. Reduction of caveolin and caveolae in oncogenically transformed cells. Proc Natl Acad Sci U S A 1995;92:1381-5.

5. Morgan MB, Stevens GL, Tannenbaum M, Salup R. Expression of the caveolins in dermal vascular tumors. J Cutan Pathol 2001;28:24-8.

6. Sadahira Y, Moriya T, Shirabe T, Matsuno T, Manabe T. Epstein-Barr virus associated post-transplant primary smooth muscle tumor of the liver: report of an autopsy case. Pathol Int 1996;46:601-4.

7. De Vos S, Wilczyski SP, Fleischhacker M, Koeffler P. p53 alterations in uterine leiomyosarcomas versus leiomyomas. Gynecol Oncol 1994;54:205-8.

8. Wang M, Xu Y, Zhang T. Smooth muscle neoplasms of the uterus-a 51 case study. Chin J Pathol 1996;25:263-5.

9. Stratton MR, Williams S, Fisher C, Ball A, Westbury G, Gusterson BA, Fletcher CD, Knight JC, Fung YK, Reeves BR. Structural alterations of the RB1 gene in human soft tissue tumors. Br J Cancer 1989;60:202-5.

10. Sreekantaiah C, Davis JR, Sandberg AA. Chromosomal abnormalities in leiomyosarcomas. Am J Pathol 1993;142:293-305.

11. Boghosian L, Dal Cin P, Turc-Carel C, Rao U, Karakousis C, Sait SJ, Sandberg AA. Three possible cytogenetic subgroups of leiomyosarcoma. Can Gen Cytogenet 1989;43:39-49.

12. Van der Heijden O, Chiu H, Park TC, Takahashi H, LiVolsi VA, Risinger JI, Barrett JC, Berchuck A, Evans AC, Behbakht K, Menzin AW, Liu PC, Benjamin I, Morgan MA, King SA, Rubin SC, Boyd J. Allelotype analysis of uterine leiomyoma: localization of a potential tumor suppressor gene to a 4-cM region of chromosome 7q. Mol Carcinog 1998;2:243-7. 
13. Willen H, Akerman M, Dal Cin P, De Wever I, Fletcher CD, Mandahl N, Mertens F, Mitelman F, Rosai J, Rydholm A, Sciot R, Tallini G, Van den Berghe H, Vanni R. Comparison of chromosomal patterns with clinical features in 165 lipomas: a report of the CHAMP study group. Can Gen Cytogenet 1998;102:46-9.

14. Dei Tos A, Doglioni C, Piccinin S, Sciot R, Furlanetto A, Boiocchi M, Dal Cin P, Maestro R, Fletcher CD, Tallini G. Coordinated expression and amplification of the MDM2, CDK4, and HMG1-C genes in atypical lipomatous tumors. J Pathol 2000;190:531-6.

15. Tallini G, Dal Cin P, Rhoden KJ, Chiappetta G, Manfioletti G, Giancotti V, Fusco A, Van den Berghe H, Sciot R. Expression of HMG1-C and HMG1(Y) in ordinary lipoma and atypical lipomatous tumors: immunohistochemical reactivity correlates with karyotypic alterations. Am J Pathol 1997;151:37-43.
16. Belge G, Caselitz J, Bonk U, Bartnitzke J. Genetic studies of differential fatty tissue tumor diagnosis. Pathologe 1997;18: $160-6$.

17. Mentzel T. Biological continuum of benign, atypical, and malignant mesenchymal neoplasms-does it exist? J Pathol 2000;190:523-5.

18. Pilotti S, Della Torre G, Lavarino C, DiPalma S, Sozzi G, Minoletti F, Rao S, Pasquini G, Azzarelli A, Rilke F, Pierotti MA. Distinct mdm2/p53 expression patterns in liposarcoma subgroups: implications for different pathogenic mechanisms. J Pathol 1997;181:14-24.

19. Buto S, Pierotti M, Tamborini E, Della Torre G, Lavarino C, Rilke F, Pilotti S. Biochemical uncovering of mdm2/ p53 complexes in liposarcomas parallels their immunohistochemical detection. Diag Mol Pathol 1999;8: 125-30.

\section{Book Review}

\section{Stocker JT, Dehner, LP, editors: Pediatric Pa- thology, 2nd Edition, 1612 pp, Baltimore, Lippincott Williams \& Wilkins, 2001 (\$32500).}

This text is a comprehensive volume on the pathology of infants and children. Like the first edition, the second edition covers all aspects of pediatric pathology, including hereditary, congenital, neoplastic, and acquired disease as well as forensics. The purpose of this text is to provide a much-needed comprehensive resource not only for full-time pediatric pathologists, but also for general pathologists who find themselves frequently called upon to function as pediatric pathologists in today's managed care environment. The editors accomplish this formidable task by enlisting the help of numerous contributing authors, many of whom are notable leaders in their respective areas of expertise.

The text is organized into two sections covering general and organ system pathology, respectively. New to the second edition are chapters on the role of the pediatric pathologist and techniques in pediatric pathology. The techniques chapter addresses issues unique to the pediatric population regarding fine needle aspiration, imaging, autopsy, and molecular diagnostics. Overall, the text contains very readable and thorough discussions of all major aspects of pathologic anatomy. Tables are used extensively and effectively to present details that may otherwise get lost in the text. Where relevant, pertinent features of embryology/developmental biology are integrated into the text.

An excellent feature is the collection of appendices at the end of the text, which provide numerous graphs and tables of anthropomorphic data for children of various gestational and postgestational ages. Another excellent feature is the extensive references for each chapter. References are both historical and current, with several chapters containing more than 500 entries. One weakness of the text is the quality of gross and microscopic photographs. Poor resolution, especially on many low-power photomicrographs, precludes adequate visualization of cellular detail and morphology. Additionally, all photographs are black and white. Nevertheless, the photographs are generally helpful in making diagnoses and illustrating points made in the text.

In summary, this edition is a well written, exceedingly thorough, unrivaled component of the pathology literature. This text will be an invaluable resource to anyone faced with the challenges of pediatric pathology.

\section{Ryan DeHaan \\ University of Kansas School of Medicine \\ Kansas City, Kansas}

